\title{
Electromagnetic Modeling of Lossy Materials with a Potential-Based Boundary Element Method
}

\author{
Shashwat Sharma, Graduate Student Member, IEEE, and Piero Triverio, Senior Member, IEEE
}

\begin{abstract}
The boundary element method (BEM) enables solving three-dimensional electromagnetic problems using a twodimensional surface mesh, making it appealing for applications ranging from electrical interconnect analysis to the design of metasurfaces. The BEM typically involves the electric and magnetic fields as unknown quantities. Formulations based on electromagnetic potentials rather than fields have garnered interest recently, for two main reasons: (a) they are inherently stable at low frequencies, unlike many field-based approaches, and (b) potentials provide a more direct interface to quantum physical phenomena. Existing potential-based formulations for electromagnetic scattering have been proposed primarily for perfect conductors. We develop a potential-based BEM formulation which can capture both dielectric and conductive losses, and accurately models the skin effect over broad ranges of frequency. The accuracy of the proposed formulation is validated through canonical and realistic numerical examples.
\end{abstract}

Index Terms-Maxwell's equations, electromagnetic potentials, boundary element method, integral equations, lossy conductors.

\section{INTRODUCTION}

$\mathbf{E}$ LECTROMAGNETIC simulation tools based on the boundary element method (BEM) [1] have gained traction in a variety of applications, ranging from antenna modeling [2] to high-speed interconnect analysis [3]-[7]. The BEM is based on a surface integral representation of Maxwell's equations, which allows three-dimensional problems to be solved in terms of quantities defined on a two-dimensional surface mesh. Many conventional full-wave BEM formulations, which take electric and magnetic fields as unknown quantities, suffer from numerical instability at very low frequencies [8]. The need to model multiscale structures at both high and low frequencies arises in applications such as the analysis of integrated circuit components. This motivates the development of broadband BEM formulations where electromagnetic potentials, rather than fields, are taken as the unknowns [9], [10]. These formulations do not rely on the coupling between electric and magnetic fields, and provide a natural interface to quantum phenomena, which is an important consideration in emerging applications such as quantum computing [11], [12]. Potential-based methods may also be well-suited for coupled electromagnetic-circuit simulations.

S. Sharma is with the Edward S. Rogers Sr. Department of Electrical \& Computer Engineering, University of Toronto, Toronto, ON, M5S 3G4 Canada, e-mail: shash.sharma@mail.utoronto.ca. P. Triverio is with the Edward S. Rogers Sr. Department of Electrical \& Computer Engineering and with the Institute of Biomedical Engineering, University of Toronto, Toronto, ON, M5S 3G4 Canada, email: piero.triverio@utoronto.ca.

This work was supported by Advanced Micro Devices, by the Natural Sciences and Engineering Research Council of Canada (Collaborative Research and Development Grants program), and by CMC Microsystems.

Manuscript received ...; revised ....
Potential-based integral equation (PIE) methods for scattering analysis have been developed primarily for perfect conductors [9], [10], [13]-[15]. For low frequencies and subwavelength structures, where PIE formulations are most sorely needed, the skin depth in a conductor may be large compared to its physical dimensions, and modeling the conductor as perfect may be inaccurate. Although magnetoquasistatic PIE formulations have been used for eddy current modeling in lossy conductors [16]-[20], these techniques do not apply in the presence of dielectric inclusions, or at high frequencies. PIE-based modeling of lossless dielectric objects was considered in [21], but mainly from a theoretical perspective, and in [22], but in the time domain. Also, the method in [21] involves a linear combination of integral equations written for adjacent materials. As in the case of analogous fieldbased formulations [23]-[25], the formulation in [21] may be inaccurate for large contrasts in material parameters of adjacent media. To the best of our knowledge, a full-wave PIE formulation for lossy penetrable materials has not been demonstrated in the frequency domain.

In this article, we devise a novel full-wave PIE formulation for electromagnetic scattering from lossy dielectrics and conductors, applicable at both low and high frequencies. The proposed formulation couples the scalar and vector potential integral equations [9] in the regions internal and external to each object. An appropriate discretization is discussed, and the accuracy of the formulation is demonstrated numerically over wide ranges of conductivity and frequency.

\section{PRoposed Formulation}

We consider time-harmonic scattering from an object occupying volume $\mathcal{V}$, with surface $\mathcal{S}$ and outward unit normal vector $\hat{n}$. Symbols $\mathcal{S}^{-}$and $\mathcal{S}^{+}$denote the internal and external sides of $\mathcal{S}$, respectively. The object has permittivity $\varepsilon$, permeability $\mu$, and conductivity $\sigma>0$. The permittivity may be complex, $\varepsilon=\varepsilon^{\prime}-j \varepsilon^{\prime \prime}$, where the imaginary part represents dielectric losses [26]. The object resides in free space, $\mathcal{V}_{0}$, with permittivity $\varepsilon_{0}$ and permeability $\mu_{0}$.

\section{A. Internal Region}

For $\vec{r} \in \mathcal{V}$, the magnetic vector and electric scalar potentials, $\vec{A}(\vec{r})$ and $\phi(\vec{r})$, respectively, can be defined via [27]

$$
\begin{aligned}
\mu \vec{H}(\vec{r}) & =\nabla \times \vec{A}(\vec{r}), \\
\vec{E}(\vec{r}) & =-j \omega \vec{A}(\vec{r})-\nabla \phi(\vec{r}),
\end{aligned}
$$

where $\omega$ is the angular frequency, $\vec{H}(\vec{r})$ is the magnetic field, and $\vec{E}(\vec{r})$ is the electric field. Using (1) and (2) in 
Maxwell's equations, it can be shown that $\vec{A}(\vec{r})$ satisfies the homogeneous Helmholtz equation [27]

$$
\nabla^{2} \vec{A}(\vec{r})+k^{2} \vec{A}(\vec{r})=0,
$$

when the Lorenz gauge

$$
\nabla \cdot \vec{A}(\vec{r})=-\mu\left(j \omega \varepsilon^{\prime}+\omega \varepsilon^{\prime \prime}+\sigma\right) \phi(\vec{r})
$$

is adopted [9], [28]. In (3), $k^{2}=-j \omega \mu\left(j \omega \varepsilon^{\prime}+\omega \varepsilon^{\prime \prime}+\sigma\right)$ is the wave number associated with the object's material. Likewise, $\phi(\vec{r})$ satisfies the Helmholtz equation [27]

$$
\left(\gamma \mu^{-1}+\sigma\right)\left[\nabla^{2} \phi(\vec{r})+k^{2} \phi(\vec{r})\right]=0,
$$

where $\gamma \triangleq\left(j \omega \varepsilon^{\prime}+\omega \varepsilon^{\prime \prime}+\sigma\right) \mu$. The use of Green's identities [29] with (3) and (5) leads to the integral equations [9]

$$
\begin{aligned}
& \mathcal{L}\left[\hat{n}^{\prime} \times \nabla^{\prime} \times \vec{A}\left(\vec{r}^{\prime}\right)\right]+\mathcal{K}\left[\hat{n}^{\prime} \times \vec{A}\left(\vec{r}^{\prime}\right)\right] \\
&+\vec{A}(\vec{r})+\gamma \mathcal{L}\left[\phi\left(\vec{r}^{\prime}\right) \hat{n}^{\prime}\right]-\nabla \mathcal{L}\left[\hat{n}^{\prime} \cdot \vec{A}\left(\vec{r}^{\prime}\right)\right]=0 \\
& \\
& \gamma \mu^{-1}\left(\mathcal{L}\left[\hat{n}^{\prime} \cdot \nabla \phi\left(\vec{r}^{\prime}\right)\right]+\mathcal{M}\left[\phi\left(\vec{r}^{\prime}\right)\right]-\phi(\vec{r})\right)=0
\end{aligned}
$$

where primed and unprimed coordinates denote source and observation points, respectively, $\vec{r} \in \mathcal{V}$, and $\vec{r}^{\prime} \in \mathcal{S}^{-}$. The integral operators in (6) and (7) are defined as [1]

$$
\begin{aligned}
\mathcal{L}\left[\vec{a}\left(\vec{r}^{\prime}\right)\right] & =\int_{\mathcal{S}} d \mathcal{S}^{\prime} G\left(k, \vec{r}, \vec{r}^{\prime}\right) \vec{a}\left(\vec{r}^{\prime}\right), \\
\mathcal{K}\left[\vec{a}\left(\vec{r}^{\prime}\right)\right] & =\int_{\mathcal{S}} d \mathcal{S}^{\prime} \nabla G\left(k, \vec{r}, \vec{r}^{\prime}\right) \times \vec{a}\left(\vec{r}^{\prime}\right), \\
\mathcal{M}\left[a\left(\vec{r}^{\prime}\right)\right] & =\int_{\mathcal{S}} d \mathcal{S}^{\prime} \hat{n}^{\prime} \cdot \nabla G\left(k, \vec{r}^{\prime}, \vec{r}^{\prime}\right) a\left(\vec{r}^{\prime}\right),
\end{aligned}
$$

where $G\left(k, \vec{r}, \vec{r}^{\prime}\right)$ is the Green's function associated with the object's material,

$$
G\left(k, \vec{r}, \vec{r}^{\prime}\right)=\frac{e^{-j k\left|\vec{r}-\vec{r}^{\prime}\right|}}{4 \pi\left|\vec{r}-\vec{r}^{\prime}\right|} .
$$

Next, the object's surface is discretized with a triangular mesh. Quantity $\hat{n} \times \nabla \times \vec{A}(\vec{r})$ is expanded with RaoWilton-Glisson (RWG) functions [30], $\vec{f}_{n}(\vec{r})$ normalized by edge length, while $\hat{n} \times \vec{A}(\vec{r})$ is expanded with BuffaChristiansen functions [31], $\vec{g}_{n}(\vec{r})$, which are defined on a barycentric refinement of the mesh. This choice of functions stems from (1) and (2), which indicate that $\hat{n} \times \nabla \times \vec{A}(\vec{r})$ and $\hat{n} \times \vec{A}(\vec{r})$ are related to $\hat{n} \times \vec{H}(\vec{r})$ and $\hat{n} \times \vec{E}(\vec{r})$ on $\mathcal{S}$, respectively. Since $\hat{n} \times \vec{H}(\vec{r})$ and $\hat{n} \times \vec{E}(\vec{r})$ can be interpreted as electric and magnetic surface current densities, respectively, $\hat{n} \times \nabla \times \vec{A}(\vec{r})$ and $\hat{n} \times \vec{A}(\vec{r})$ must both be expanded with divergence-conforming basis functions [32], while respecting their mutual orthogonality [33]. These requirements are satisfied by the proposed expansion scheme.

Unknowns $\phi(\vec{r})$ and $\hat{n} \cdot \vec{A}(\vec{r})$ are expanded with unitamplitude pulse functions, $h_{n}(\vec{r})$, while $\hat{n} \cdot \nabla \phi(\vec{r})$ is expanded with area-normalized pulse functions, $\widetilde{h}_{n}(\vec{r})$. The choice of normalizing by area was based on an empirical study of the condition number of the final system matrix. A more sophisticated choice of basis function for $\phi(\vec{r})$, such as the one suggested in [22], may lead to improved accuracy. However, as shown in Section III, the pulse functions are sufficiently accurate in a variety of cases.

Taking the cross product of $\hat{n}$ with (6) and letting $\vec{r} \rightarrow \mathcal{S}^{-}$ gives its rotated tangential part, which is tested with $\hat{n} \times \mathrm{RWG}$ functions to get the matrix relation

$$
\begin{aligned}
\mathbf{L}^{(\mathrm{ff})} \mathbf{a}_{c,}+\left(\mathbf{K}^{(\mathrm{fg})}+\right. & \left.\frac{1}{2} \mathbf{I}_{\times}^{(\mathrm{fg})}\right) \mathbf{a}_{t,} \\
& +\gamma \mathbf{L}^{(\mathrm{fh})} \mathbf{\Phi}+\mathbf{D}^{T} \mathbf{L}^{(\mathrm{hh})} \mathbf{a}_{n}=\mathbf{0} .
\end{aligned}
$$

Taking the dot product of $\hat{n}$ with (6) and letting $\vec{r} \rightarrow \mathcal{S}^{-}$gives its normal component, which is tested with $\widetilde{h}_{n}(\vec{r})$ to obtain

$$
\begin{aligned}
\mathbf{L}^{(\mathrm{hf})} \mathbf{a}_{c,}+\mathbf{K}^{(\mathrm{hg})} \mathbf{a}_{t,}+\gamma \mathbf{L}^{(\mathrm{hh})} \mathbf{\Phi} & \\
& -\left(-\left(\mathbf{A} \mathbf{I}^{(\mathrm{hh})}\right)^{T}-\frac{1}{2} \mathbf{I}_{\|}^{(\mathrm{hh})}\right) \mathbf{a}_{n},=\mathbf{0},
\end{aligned}
$$

where the superscript " $T$ " denotes the matrix transpose. Finally, taking $\vec{r} \rightarrow \mathcal{S}^{-}$in (7) and testing it with $\widetilde{h}_{n}(\vec{r})$ yields

$$
\gamma \mu^{-1}\left[\mathbf{L}^{(\mathrm{hh})} \mathbf{\Psi}+\left(\mathbf{A}^{(\mathrm{hh})}-\frac{1}{2} \mathbf{I}_{\|}^{(\mathrm{hh})}\right) \boldsymbol{\Phi}\right]=\mathbf{0} .
$$

In (12), (13), and (14), $\mathbf{L}, \mathbf{K}$ and $\mathbf{M}$ are the discretized $\mathcal{L}, \mathcal{K}$ and $\mathcal{M}$ operators, respectively, where a dash through a matrix indicates that the associated integral is computed in the principal value sense [1]. Term $\mathbf{D}$ is a sparse incidence matrix linking mesh edges and triangles [34]. Identity operator $\mathbf{I}_{\times}^{(\mathrm{fg})}$ is obtained by testing $\vec{g}_{n}(\vec{r})$ with $\hat{n} \times \vec{f}_{n}(\vec{r})$, while $\mathbf{I}_{\|}^{(\mathrm{hh})}$ involves testing $h_{n}(\vec{r})$ with $\widetilde{h}_{n}(\vec{r})$. The superscript labels $(i j)$ on each discrete operator represent the testing and basis functions involved, respectively. Column vectors $\mathbf{a}_{c}, \mathbf{a}_{t}, \mathbf{a}_{n}, \boldsymbol{\Phi}$ and $\boldsymbol{\Psi}$ contain the unknown coefficients associated with $\hat{n} \times \nabla \times \vec{A}(\vec{r}), \hat{n} \times \vec{A}(\vec{r}), \hat{n} \cdot \vec{A}(\vec{r}), \phi(\vec{r})$ and $\hat{n} \cdot \nabla \phi(\vec{r})$, respectively.

\section{B. External Region}

Next, PIEs are devised to capture the physics in the region external to $\mathcal{S}$, following the procedure in Section II-A for $\vec{r}, \vec{r}^{\prime} \in \mathcal{S}^{+}$. However, instead of using the normal component of (6) for the external region, we take its divergence [13] and test the resulting equation with $\widetilde{h}_{n}(\vec{r})$. We found that this choice leads to better conditioning of the final system matrix, and better accuracy. The resulting discrete equations are

$$
\begin{aligned}
& \mathbf{L}_{0}^{(\mathrm{ff})} \mathbf{a}_{c, 0}+\left(\mathbf{K}_{0}^{(\mathrm{fg})}-\frac{1}{2} \mathbf{I}_{\times}^{(\mathrm{fg})}\right) \mathbf{a}_{t, 0} \\
&+\gamma_{0} \mathbf{L}_{0}^{(\mathrm{fh})} \mathbf{\Phi}_{0}+\mathbf{D}^{T} \mathbf{L}_{0}^{(\mathrm{hh})} \mathbf{a}_{n, 0}=-\mathbf{a}_{t, \mathrm{inc}}, \\
& \mathbf{L}_{0}^{(\mathrm{hh})} \mathbf{D} \mathbf{a}_{c, 0}+\gamma_{0}\left(\mathbf{A}_{0}^{(\mathrm{hh})}+\frac{1}{2} \mathbf{I}_{\|}^{(\mathrm{hh})}\right) \boldsymbol{\Phi}_{0} \\
&+k_{0}^{2} \mathbf{L}_{0}^{(\mathrm{hh})} \mathbf{a}_{n, 0}=\gamma_{0} \boldsymbol{\Phi}_{\mathrm{inc}}, \\
& \mathbf{L}_{0}^{(\mathrm{hh})} \mathbf{\Psi}_{0}+\left(\mathbf{M}_{0}^{(\mathrm{hh})}+\frac{1}{2} \mathbf{I}_{\|}^{(\mathrm{hh})}\right) \mathbf{\Phi}_{0}=-\boldsymbol{\Phi}_{\mathrm{inc}},
\end{aligned}
$$

where the subscript "0" on the matrix operators denotes that the Green's function associated with $\mathcal{V}_{0}$ is used, and $\gamma_{0} \triangleq j \omega \varepsilon_{0} \mu_{0}$. Subscript " 0 " on the column vectors of unknowns indicates that the quantities are defined on $\mathcal{S}^{+}$. Subscript "inc" indicates incident potentials [9], [10]. 


\section{Boundary Conditions}

For $\hat{n} \times \nabla \times \vec{A}(\vec{r}), \hat{n} \times \vec{A}(\vec{r})$ and $\phi(\vec{r})$, we use boundary conditions on $\mathcal{S}$ identical to those in [9], [10],

$$
\begin{aligned}
\mu_{0}^{-1} \hat{n} \times \nabla \times \vec{A}_{0}(\vec{r}) & =\mu^{-1} \hat{n} \times \nabla \times \vec{A}(\vec{r}), \\
\hat{n} \times \vec{A}_{0}(\vec{r}) & =\hat{n} \times \vec{A}(\vec{r}), \\
\phi_{0}(\vec{r}) & =\phi(\vec{r}),
\end{aligned}
$$

where $\vec{A}(\vec{r})$ and $\vec{A}_{0}(\vec{r})$ are the magnetic vector potentials on $\mathcal{S}^{-}$and $\mathcal{S}^{+}$, respectively. For conductive objects, a new boundary condition is required for $\hat{n} \cdot \vec{A}(\vec{r})$ and $\hat{n} \cdot \nabla \phi(\vec{r})$,

$$
\begin{aligned}
-j \omega & {\left[\gamma_{0} \mu_{0}^{-1} \hat{n} \cdot \vec{A}_{0}(\vec{r})-\gamma \mu^{-1} \hat{n} \cdot \vec{A}(\vec{r})\right] } \\
& -\left[\gamma_{0} \mu_{0}^{-1} \hat{n} \cdot \nabla \phi_{0}(\vec{r})-\gamma \mu^{-1} \hat{n} \cdot \nabla \phi(\vec{r})\right]=0,
\end{aligned}
$$

which is used to eliminate $\hat{n} \cdot \nabla \phi(\vec{r})$ in (7). Equation (21) is derived using (2), (4) and the standard boundary conditions [28] for $\hat{n} \cdot \vec{E}(\vec{r}), \hat{n} \cdot \vec{E}_{0}(\vec{r})$ and $\hat{n} \cdot \vec{J}(\vec{r})$, where $\vec{E}(\vec{r})$ and $\vec{E}_{0}(\vec{r})$ are the electric fields in $\mathcal{V}$ and $\mathcal{V}_{0}$, respectively, and $\vec{J}(\vec{r})$ is the conduction volume current density in $\mathcal{V}$.

\section{Final System Matrix}

Equations (15), (12), (16), (13), (14) and (17) are concatenated in that order, and the boundary condition (21) is applied to get the final system of equations (22) at the top of the following page, where $\xi$ is the average mesh edge length. In (22), the equations and unknowns have been strategically scaled to ensure stable direct factorization and good accuracy for wide ranges of frequency and conductivity. It may be necessary to enforce charge conservation at extremely low frequencies. Although this point is not addressed in the present formulation, the results in Section III demonstrate the extremely wide ranges of frequency over which the proposed method still remains accurate. Also, the system matrix in (22) contains two vector quantities $\left(\mathbf{a}_{c, 0}, \mathbf{a}_{t,}\right)$ and four scalar quantities ( $\left.\boldsymbol{\Phi}, \mathbf{a}_{n}, \mathbf{a}_{n, 0} \boldsymbol{\Psi}_{0}\right)$ as unknowns, while most fieldbased formulations for lossy conductors contain only two vector and up to one scalar unknown quantity [3], [7], [35]. However, the additional unknowns in the proposed method may be well worth the broadband performance, particularly when coupled with acceleration algorithms [36]-[39]. It may be possible to obtain a smaller system matrix, but the scope of this work is to establish that potential-based formulations can be used to model lossy conductors accurately and over very wide ranges of frequency and conductivity.

\section{RESULTS}

The accuracy of the proposed formulation is validated through comparisons with analytical solutions and an existing field-based BEM formulation: the enhanced augmented electric field integral equation (eAEFIE) for penetrable objects [35], [40]. The numerical integration routines in [3] were used for the operators associated with the internal region, to maintain accuracy for highly conductive media. Electric and magnetic fields tangential to $\mathcal{S}$ were obtained as a postprocessing step via (1) and (2).

\section{A. Sphere}

First, we consider a sphere with diameter $1 \mathrm{~m}$ and relative permittivity 2 , excited by a plane wave. The sphere is meshed with 2,114 triangles, and the bistatic radar cross section (RCS) is compared against the analytical Mie series (Fig. 1). The RCS is reported for the plane along which the incident electric field is polarized ( $E$-plane). We consider conductivities spanning 10 decades from $10^{-3} \mathrm{~S} / \mathrm{m}$, where the sphere behaves like a dielectric, to $10^{7} \mathrm{~S} / \mathrm{m}$, corresponding to a good conductor. Nine decades of frequencies from $1 \mathrm{~Hz}$ to $1 \mathrm{GHz}$ are simulated to encompass skin depths from $1 \mu \mathrm{m}$ to several times the sphere's diameter. For the $1 \mathrm{GHz}$ cases, a finer mesh with 3,786 triangles was used. Fig. 1 demonstrates the excellent accuracy of the proposed formulation. The results deviate slightly from the Mie series in the bottom panel, but identical deviations are observed for the eAEFIE formulation. Therefore, these errors can be attributed to the discretization and numerical integration, which are common to both formulations.

\section{B. Cube}

Next, we consider an FR-4 cube meshed with 3,274 triangles, with side length $1 \mathrm{~m}, \varepsilon^{\prime}=4.4 \varepsilon_{0}$, and loss tangent $\tan \delta=\varepsilon^{\prime \prime} / \varepsilon^{\prime}=0.03$ [41]. A plane wave impinges on the cube with the electric field polarized as shown in the inset in the bottom panel of Fig. 2. The E-plane bistatic RCS for the proposed formulation is compared with the results obtained via the eAEFIE, for frequencies between $10 \mathrm{kHz}$ and $1 \mathrm{GHz}$. The top panel of Fig. 2 demonstrates the excellent accuracy of the proposed formulation compared to the eAEFIE. We also considered the case when $\varepsilon^{\prime}=\varepsilon_{0}, \varepsilon^{\prime \prime}=0$, and $\sigma \in\left[10^{-3}, 10^{7}\right] \mathrm{S} / \mathrm{m}$ at $100 \mathrm{MHz}$, corresponding to skin depths between $1.6 \mathrm{~m}$ and $16 \mu \mathrm{m}$. Excellent agreement with the eAEFIE was achieved, as shown in the bottom panel of Fig. 2 . The inset shows the surface current density for $\sigma=10 \mathrm{~S} / \mathrm{m}$.

\section{Split Ring Resonator Array}

Finally, we consider a $2 \times 2$ array of split ring resonators (SRRs), which are of relevance in the design of metamaterials and metasurfaces [42]. Each element has a relative permittivity of 11 and an electrical conductivity of $10^{7} \mathrm{~S} / \mathrm{m}$. The elements have side length $2 \mu \mathrm{m}$, width $0.2 \mu \mathrm{m}$, and height $0.1 \mu \mathrm{m}$. The width of the gap is $0.2 \mu \mathrm{m}$. The structure is meshed with 3,392 triangles, and excited with an incident plane wave traveling along the $-z$ direction, with the electric field polarized in the $y$ direction. The geometry is shown in Fig. 3. We consider the frequencies $10 \mathrm{GHz}, 1 \mathrm{THz}$ and $100 \mathrm{THz}$. Fig. 3 shows the electric surface current density for the $100 \mathrm{THz}$ case. Fig. 4 shows the magnitude of the electric field measured along the probe line shown in Fig. 3. The probe line is placed along the $x$ axis in the $x z$ plane bisecting the array, $0.4 \mu \mathrm{m}$ above it. Excellent agreement is obtained compared to the eAEFIE for all three frequencies, demonstrating the accuracy of the proposed PIE formulation for realistic structures.

\section{CONCLUSION}

A boundary element formulation based on the electric scalar and magnetic vector potential is proposed for the accurate 


$$
\left[\begin{array}{cccccc}
\frac{1}{\xi} \mathbf{L}_{0}^{(\mathrm{ff})} & \mathbf{K}_{0}^{(\mathrm{fg})}-\frac{1}{2} \mathbf{I}_{\times}^{(\mathrm{fg})} & \frac{j k_{0}}{\xi} \mathbf{L}_{0}^{(\mathrm{fh})} & \mathbf{0} & \frac{1}{\xi} \mathbf{D}^{T} \mathbf{L}_{0}^{(\mathrm{hh})} & \mathbf{0} \\
\frac{\mu}{\xi \mu_{0}} \mathbf{L}^{(\mathrm{ff})} & \mathbf{K}^{(\mathrm{fg})}+\frac{1}{2} \mathbf{I}_{\times}^{(\mathrm{fg})} & \frac{c_{0} \gamma}{\xi} \mathbf{L}^{(\mathrm{fh})} & \frac{1}{\xi} \mathbf{D}^{T} \mathbf{L}^{(\mathrm{hh})} & \mathbf{0} & \mathbf{0} \\
\mathbf{L}_{0}^{(\mathrm{hh})} \mathbf{D} & \mathbf{0} & j k_{0}\left(\mathbf{I}_{0}^{(\mathrm{hh})}+\frac{1}{2} \mathbf{I}_{\|}^{(\mathrm{hh})}\right. & \mathbf{0} & k_{0}^{2} \mathbf{L}_{0}^{(\mathrm{hh})} & \mathbf{0} \\
\frac{\mu}{\mu_{0}} \mathbf{L}^{(\mathrm{hf})} & \xi \mathbf{K}^{(\mathrm{hg})} & c_{0} \gamma \mathbf{L}^{(\mathrm{hh})} & \left(\mathbf{M} \mathbf{I}^{(\mathrm{hh})}\right)^{T}-\frac{1}{2} \mathbf{I}_{\|}^{(\mathrm{hh})} & \mathbf{0} & \mathbf{0} \\
\mathbf{0} & \mathbf{0} & \mathbf{M}^{(\mathrm{hh})}-\frac{1}{2} \mathbf{I}_{\|}^{(\mathrm{hh})} & \frac{k^{2}}{\gamma c_{0}} \mathbf{L}^{(\mathrm{hh})} & -\frac{k_{0}^{2}}{\gamma c_{0}} \frac{\mu}{\mu_{0}} \mathbf{L}^{(\mathrm{hh})} & \frac{\gamma}{\gamma c_{0}} \frac{\mu}{\mu_{0}} \mathbf{L}^{(\mathrm{hh})} \\
\mathbf{0} & \mathbf{0} & \mathbf{M}_{0}^{(\mathrm{hh})}-\frac{1}{2} \mathbf{I}_{\|}^{(\mathrm{hh})} & \mathbf{0} & \mathbf{0} & \frac{1}{c_{0}} \mathbf{L}_{0}^{(\mathrm{hh})}
\end{array}\right]\left[\begin{array}{c}
\mathbf{a}_{c, 0} \\
\mathbf{a}_{t,} / \xi \\
\mathbf{\Phi} / c_{0} \\
\mathbf{a}_{n,} \\
\mathbf{a}_{n, 0} \\
\mathbf{\Psi}_{0}
\end{array}\right]\left[\begin{array}{c}
-\mathbf{a}_{t, \text { inc }} / \xi \\
\mathbf{0} \\
\gamma_{0} \mathbf{\Phi}_{\mathrm{inc}} \\
\mathbf{0} \\
\mathbf{0} \\
-\boldsymbol{\Phi}_{\mathrm{inc}} / c_{0}
\end{array}\right]
$$
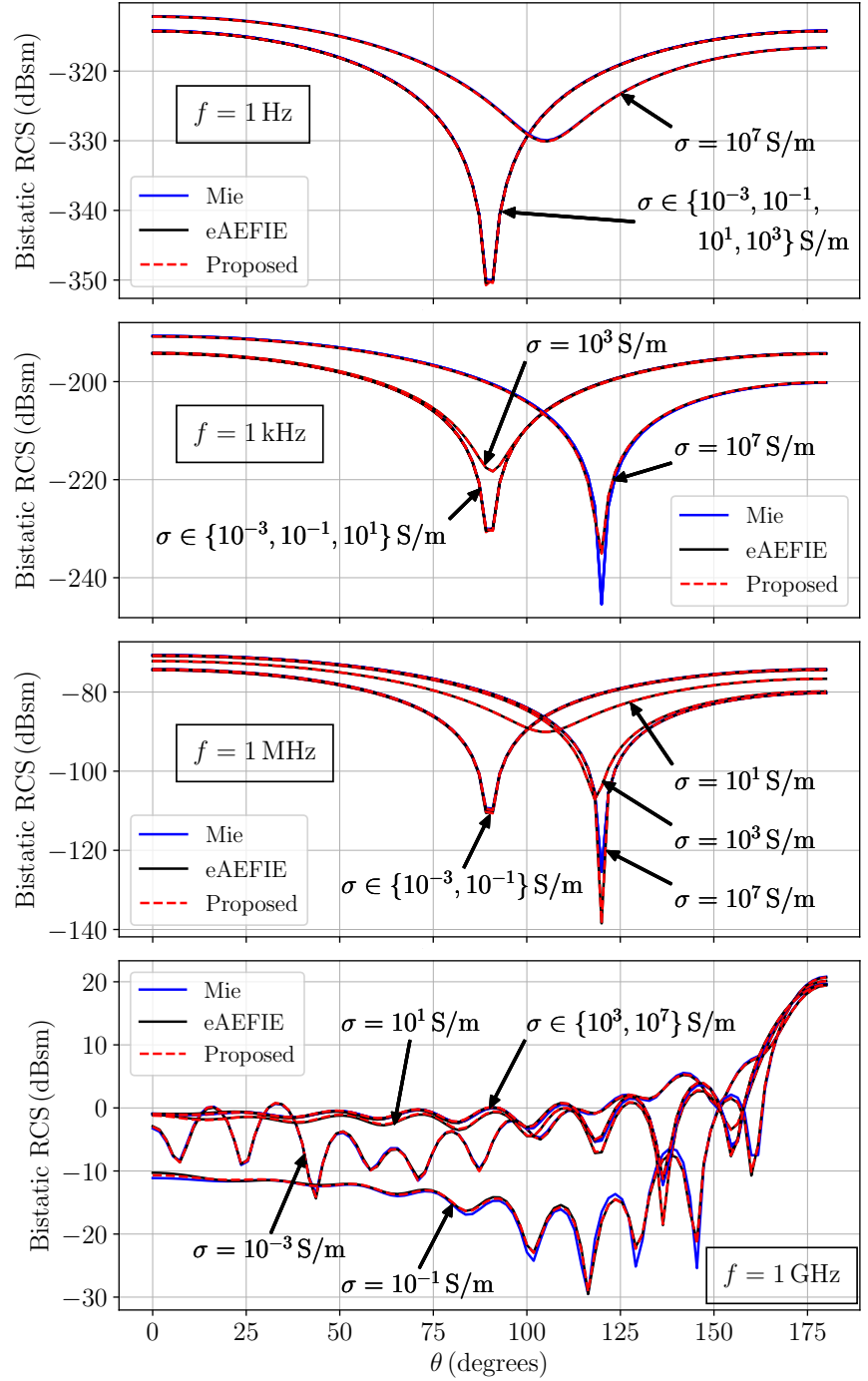

Fig. 1: Accuracy validation for the sphere in Section III-A. The same horizontal axis applies to all panels.

modeling of lossy objects over wide ranges of frequency and conductivity. Unlike existing potential-based scattering formulations, the proposed method accurately captures the skin effect both at low and high frequencies, and can model both good conductors and lossy dielectrics. The accuracy of the proposed formulation is validated through canonical and realistic numerical examples, and excellent agreement with analytical results and an existing field-based method is observed for at least nine decades of frequency and conductivity.

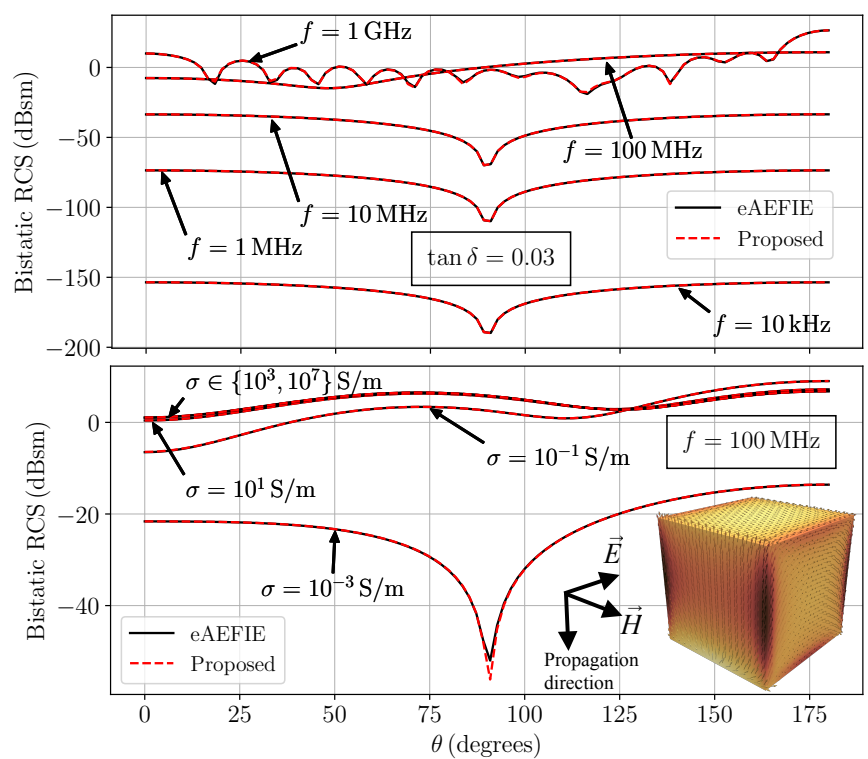

Fig. 2: Accuracy validation for the cube in Section III-B.

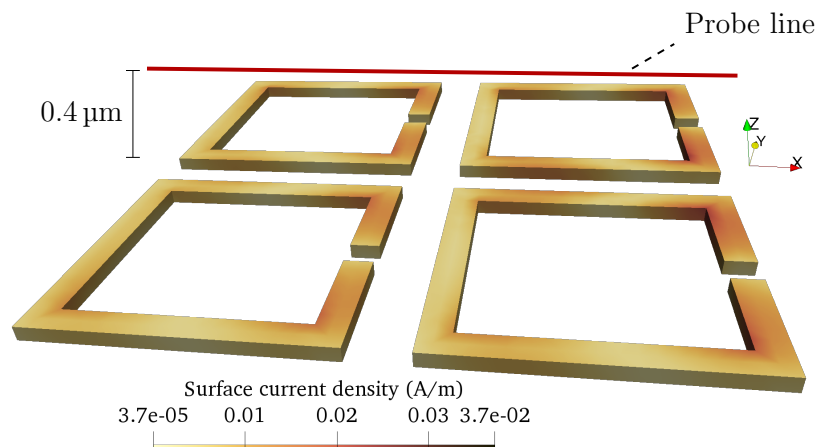

Fig. 3: Geometry and electric surface current density magnitude for the SRR array in Section III-C.

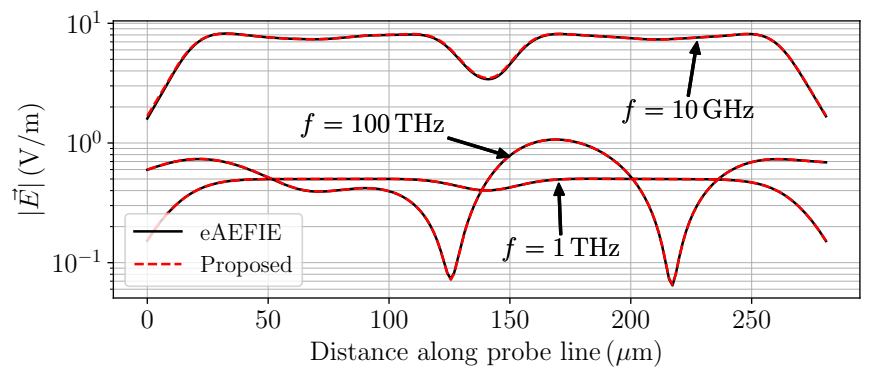

Fig. 4: Near-field $|\vec{E}(\vec{r})|$ for the SRR array in Section III-C. 


\section{REFERENCES}

[1] W. C. Chew, Waves and Fields in Inhomogeneous Media. Hoboken, NJ, USA: Wiley, 1999

[2] P. Yla-Oijala, M. Taskinen, and J. Sarvas, "Surface integral equation method for general composite metallic and dielectric structures with junctions," Prog. Electromagn. Res., vol. 52, pp. 81-108, 2005.

[3] Z. G. Qian, W. C. Chew, and R. Suaya, "Generalized impedance boundary condition for conductor modeling in surface integral equation," IEEE Trans. Microw. Theory Tech., vol. 55, no. 11, pp. 2354-2364, Nov. 2007.

[4] W. Chai and D. Jiao, "Direct matrix solution of linear complexity for surface integral-equation-based impedance extraction of complicated 3 D structures," Proc. IEEE, vol. 101, no. 2, pp. 372-388, Jun. 2013.

[5] M. Huynen, K. Y. Kapusuz, X. Sun, G. Van der Plas, E. Beyne, D. De Zutter, and D. Vande Ginste, "Entire domain basis function expansion of the differential surface admittance for efficient broadband characterization of lossy interconnects," IEEE Trans. Microw. Theory Tech., vol. 68, no. 4, pp. 1217-1233, Jan. 2020.

[6] S. Sharma and P. Triverio, "An accelerated surface integral equation method for the electromagnetic modeling of dielectric and lossy objects of arbitrary conductivity," IEEE Trans. Antennas Propag., vol. 69, no. 9, pp. 5822-5836, Sep. 2021.

[7] —, "SLIM: A well-conditioned single-source boundary element method for modeling lossy conductors in layered media," IEEE Antennas Wireless Propag. Lett., vol. 19, no. 12, pp. 2072-2076, Sep. 2020.

[8] Z. G. Qian and W. C. Chew, "A quantitative study on the low frequency breakdown of EFIE," Microw. Opt. Technol. Lett., vol. 50, no. 5, pp. $1159-1162,2008$

[9] W. C. Chew, "Vector potential electromagnetics with generalized gauge for inhomogeneous media: Formulation," Prog. Electromagn. Res., vol. 149, pp. 69-84, Sep. 2014.

[10] F. Vico, M. Ferrando, L. Greengard, and Z. Gimbutas, "The decoupled potential integral equation for time-harmonic electromagnetic scattering," Commun. Pure Appl. Math., vol. 69, no. 4, pp. 771-812, 2016.

[11] W. C. Chew, A. Y. Liu, C. Salazar-Lazaro, and W. E. I. Sha, "Quantum electromagnetics: A new look - part I," IEEE J. Multiscale Multiphys. Comput. Tech., vol. 1, pp. 73-84, Oct. 2016.

[12] — "Quantum electromagnetics: A new look - part II," IEEE $J$. Multiscale Multiphys. Comput. Tech., vol. 1, pp. 85-97, Oct. 2016.

[13] Q. S. Liu, S. Sun, and W. C. Chew, "A potential-based integral equation method for low-frequency electromagnetic problems," IEEE Trans. Antennas Propag., vol. 66, no. 3, pp. 1413-1426, Mar. 2018.

[14] U. M. Gur and O. Ergul, "Accuracy of sources and near-zone fields when using potential integral equations at low frequencies," IEEE Antennas Wireless Propag. Lett., vol. 16, pp. 2783-2786, Aug. 2017.

15] T. E. Roth and W. C. Chew, "Development of stable A- $\Phi$ time-domain integral equations for multiscale electromagnetics," IEEE J. Multiscale Multiphys. Comput. Tech., vol. 3, pp. 255-265, Dec. 2018.

[16] C. Emson and J. Simkin, "An optimal method for 3-D eddy currents," IEEE Trans. Magn., vol. 19, no. 6, pp. 2450-2452, Nov. 1983.

[17] T. Morisue and M. Fukumi, "3-D eddy current calculations using the magnetic vector potential," IEEE Trans. Magn., vol. 24, no. 1, pp. 106109, Jan. 1988.

[18] T. Morisue, "A new formulation of the magnetic vector potential method in 3-D multiply connected regions," IEEE Trans. Magn., vol. 24, no. 1, pp. 110-113, Jan. 1988.

[19] H. Tsuboi and M. Tanaka, "Three-dimensional eddy current analysis by the boundary element method using vector potential," IEEE Trans. Magn., vol. 26, no. 2, pp. 454-457, Mar. 1990.

[20] C. Bryant, C. Emson, and C. Trowbridge, "A general purpose 3D formulation for eddy currents using the Lorentz gauge," IEEE Trans. Magn., vol. 26, no. 5, pp. 2373-2375, Sep. 1990.

[21] J. Li, X. Fu, and B. Shanker, "Decoupled potential integral equations for electromagnetic scattering from dielectric objects," IEEE Trans. Antennas Propag., vol. 67, no. 3, pp. 1729-1739, Mar. 2019.
[22] T. E. Roth and W. C. Chew, "Lorenz gauge potential-based time domain integral equations for analyzing subwavelength penetrable regions," IEEE J. Multiscale Multiphys. Comput. Tech., vol. 6, pp. 24-34, Feb. 2021.

[23] A. Poggio and E. Miller, "Integral equation solutions of threedimensional scattering problems," in Computer Techniques for Electromagnetics, ser. International Series of Monographs in Electrical Engineering. Pergamon, 1973, pp. $159-264$.

[24] Y. Chang and R. Harrington, "A surface formulation for characteristic modes of material bodies," IEEE Trans. Antennas Propag., vol. 25, no. 6 , pp. 789-795, Nov. 1977.

[25] T. Wu and L. L. Tsai, "Scattering from arbitrarily-shaped lossy dielectric bodies of revolution," Radio Sci., vol. 12, no. 5, pp. 709-718, Sep. 1977.

[26] D. M. Pozar, Microwave Engineering, 4th ed. Hoboken, NJ, USA Wiley, 2012

[27] J. D. Jackson, Classical Electrodynamics, 3rd ed. Hoboken, NJ, USA: Wiley, 1999.

[28] E. J. Rothwell and M. J. Cloud, Electromagnetics, 3rd ed. Boca Raton, FL, USA: CRC Press, 2018.

[29] G. W. Hanson and A. B. Yakovlev, Operator Theory for Electromagnetics. New York, NY, USA: Springer-Verlag, 2002.

[30] S. Rao, D. Wilton, and A. Glisson, "Electromagnetic scattering by surfaces of arbitrary shape," IEEE Trans. Antennas Propag., vol. 30, no. 3, pp. 409-418, May 1982.

[31] A. Buffa and S. H. Christiansen, "A dual finite element complex on the barycentric refinement," Math. Computation, vol. 76, pp. 1743-1769, 2007.

[32] W. Chew, M. Tong, and B. Hu, Integral Equation Methods for Electromagnetic and Elastic Waves. San Rafael, CA, USA: Morgan \& Claypool, 2008.

[33] F. P. Andriulli, K. Cools, H. Bagci, F. Olyslager, A. Buffa, S. Christiansen, and E. Michielssen, "A multiplicative Calderon preconditioner for the electric field integral equation," IEEE Trans. Antennas Propag., vol. 56, no. 8, pp. 2398-2412, Aug. 2008.

[34] Z.-G. Qian and W. C. Chew, "Fast full-wave surface integral equation solver for multiscale structure modeling," IEEE Trans. Antennas Propag., vol. 57, no. 11, pp. 3594-3601, Nov. 2009.

[35] T. Xia, H. Gan, M. Wei, W. C. Chew, H. Braunisch, Z. Qian, K. Aygün, and A. Aydiner, "An integral equation modeling of lossy conductors with the enhanced augmented electric field integral equation," IEEE Trans. Antennas Propag., vol. 65, no. 8, pp. 4181-4190, Aug. 2017.

[36] J. Song, C.-C. Lu, and W. C. Chew, "Multilevel fast multipole algorithm for electromagnetic scattering by large complex objects," IEEE Trans. Antennas Propag., vol. 45, no. 10, pp. 1488-1493, Oct. 1997.

[37] E. Bleszynski, M. Bleszynski, and T. Jaroszewicz, "AIM: Adaptive integral method for solving large-scale electromagnetic scattering and radiation problems," Radio Sci., vol. 31, no. 5, pp. 1225-1251, Sep. 1996.

[38] Z. Zhu, B. Song, and J. K. White, "Algorithms in FastImp: a fast and wide-band impedance extraction program for complicated 3-D geometries," IEEE Trans. Comput.-Aided Design Integr. Circuits Syst., vol. 24, no. 7, pp. 981-998, Jul. 2005.

[39] S. Sharma and P. Triverio, "AIMx: An extended adaptive integral method for the fast electromagnetic modeling of complex structures," IEEE Trans. Antennas Propag., 2021 (early access).

[40] T. Xia, H. Gan, M. Wei, W. C. Chew, H. Braunisch, Z. Qian, K. Aygün, and A. Aydiner, "An enhanced augmented electric-field integral equation formulation for dielectric objects," IEEE Trans. Antennas Propag., vol. 64, no. 6, pp. 2339-2347, Jun. 2016.

[41] A. Djordjevic, R. Biljie, V. Likar-Smiljanic, and T. Sarkar, "Wideband frequency-domain characterization of FR-4 and time-domain causality," IEEE Trans. Electromagn. Compat., vol. 43, no. 4, pp. 662-667, 2001.

[42] D. Güney, T. Koschny, and C. M. Soukoulis, "Reducing ohmic losses in metamaterials by geometric tailoring," Phys. Rev. B, vol. 80, p. 125129 , Sep. 2009 